\title{
A URL Shortening Service by Using Flask Framework Based on Base-62 Algorithm
}

\author{
B. Suresh Babu', Thota Ravisankar ${ }^{2}$, Sriharsha ${ }^{3}$ \\ ${ }^{1-3}$ Department of H\&S, Sreyas Institute of Engineering and Technology \\ bsureshmaths@gmail.com
}

\begin{abstract}
In this paper, we have shown the importance of URL shortening at the time of sharing on various platforms. URLs appear to be long, unattractive, on most of the social platforms. They often get broken when shared on social media platforms like e-mail and short URLs can come in handy during these times. To shorten an Internet Long URL, we proposed a URL shortening service that will take a long Web URL/address and creates a shorter URL that will not break when we share on different platforms and make them more manageable by using flask framework base62 algorithm. In this paper, we have also provided a way that helps during digital marketing, social campaigning, and posting by giving the stats of the number of clicks made to the shortened URL as it shows the number of people interested in clicking the URL.
\end{abstract}

Keywords: URL Shortening, Data Flow Architecture, SQLite database engine, URL Redirection, Tracking

\section{Introduction}

An organization that works everything to do with links, URL shortener is something that we use quite a lot. With the presence of social media networks where the text are required to fit in a restricted number of characters usually to 140 characters, therefore shortening of URLs became more and more crucial. So, to shorten the long URLs, a greater number of URL shortening services are being used [1]. When somebody needs to shorten their ugly long URL they go to a URL shortening service and submit the long URL intended to get shortened [2]. These shortened URLs are not typically longer than 30 to 40 characters. These shortened URLs are then used instead of the long URLs to visit the respective websites.

In this paper, we have shown how we provide a custom URL shortening service. A custom URL shortener is connecting our custom domain to a URL shortener which acts as the foundation for all the short links that we create. Instead of a generic domain, we are combining the shortened one with our custom domain [3]. This custom URL shortening service is recommended because through the custom domain while sharing the links online, it leads to increased trust in the clients for the link, greater awareness to the brand, and increases click-through rate. For example, a person may have the following complex links, which is not understandable and looks ugly such as: 
https://privatebanking.mybank.com/privatebanking/ebankver2/secure/customersupport.as px? messageID=3324341\&Sess=asp04\&passwordvalidate=true $\&$ changepassword $=$ true The shortened URL might look like this: http://domain.com/url/a23bcd7 The proposed URL shortening service will take the long URL link and gives a shorter link that will not break while sharing on social media platforms and also provides with the custom domain and by this we aim at user trust to use the service with availability.

\section{Literature Review}

In this section we have provided a brief overview of different security standards applicable to URL shortening services researched and mentioned under security threats of URL shortening in [4-5]. As the focus is more on shortening of URLs we will be referring the security standards as suitable to the research. The studies and research shows that the security is not only meant for the shortened URL but also to the users using the service. Many times we forget the question that, are people really interested in using these shortened URLs, if they are not what might be the reason behind it. When analyzed, it shows that these shortened URLs tend to be malicious, and cause spams, phishing etc. People tend to not trust the services which are malicious and not authorized for usage. Sharing the same kind of URLs, ending up on a different and irrelevant sites on redirection when the URL is clicked, and blocking the usage are few among the various security threats which result in data breach and thus resulting in issues [6].

Various studies and analyses of different URL shortening websites show that the shortening of URL is done and aimed at only reducing the amount of space that they take up when written or while sharing, but not at the URL that the user submits to shorten or at the amount of time taken to redirect to the original address [7-10]. It shows that the URL submitted for shortening might be malicious and might go unrecognized while shortening and thus resulting in sharing of the same malicious one to many more other users causing inconvenience and resulting in the less usage of the service and reducing trust among users. In our proposed URL shortening service we are trying to provide a way such that it checks the users who should prove their identity before submitting any kind of URLs and in an indirect way checking the authenticity of the URLs. We also try to provide a customized domain which shows the user name in the shortened URL because many markets depend on brand value and loyal customers which means when a person sees the URL, if he recognizes the domain and has a trust on clicking the URL, he tends the use the service more often.

The main aspect here is to show that there has to be some kind of awareness among the users for trusting the application and increasing the usage of URL shortening services. The current state of the above overview shows that we have to deal with security problems from the point of view of the normal users. Using the existing knowledge of authenticating the users and various hashing techniques we tried to propose a service that includes the customized domain thus aiming at user trust and also trying to improve the dependability of both secure shortened URL and secure user for the future scope and analysis. In this 
section, we have discussed how the proposed URL shortening service directs the research to various fields such as transformation into social media services, insights or tracking, professionalism. These fields will be hugely benefitted because of the usage of the URL shortening service [11].

\subsection{Transformation into social media services}

From the URL Shortening services we can gather huge amount of data about how many people are really interested in clicking them and we can also whether the clicks are made by any automated machine or by human himself [12]. The way these services are used can enhanced and be transformed into social media service for campaigning purposes.URL shorteners, in their way work as clusters of information. This can lead to some useful innovations in sharing links.

\subsection{Tracking}

The main benefit that we are aiming through this application is link tracking. By tracking the clicks we can tell which post got more attention and which region is interested in that particular information. By using this link tracking, the digital marketers can benefit in efficient way and by targeting the users at areas where the response is huge [13]. This type of information can be gathered through this application.

\subsection{Expertness}

When the clients are getting used to the content shared in the form of shortened links, they start feeling more comfortable to share the links and might not use or click the URL, if we share a URL that hasn't been compressed. Long-form URLs can appear ugly and appear cleaner when shortened thus increasing the usage of more URL shortening services.

\section{Proposed Framework for URL Shortener}

The frameworks required are data flow architecture which shows the flow of data from one entity to another, flask which is used for the web development, SQLite database engine for the database operations. These frameworks complete the interface and operation of the proposed service.

\subsection{Architecture for the proposed URL Shortening Service}

The architecture that we have used for the URL shortening service is data flow architecture. In data flow architecture, the data and operations are independent of each other and there are transformations of data among components. In this architecture, the data enters the system and flows through the components and finally we get some kind of output. The main objective to use this architecture is to achieve reusability and modifiability. We use the pipe and filter architecture that comes under data flow architecture. This architecture provides the incremental transformation of data by successive components. Here, the system is divided into components such as, filters, pipes, etc. The filters process the data of one form to another and pipes move a data stream from 
one filter to another. This provides concurrency and high throughput, flexibility, and supports both sequential and parallel execution.

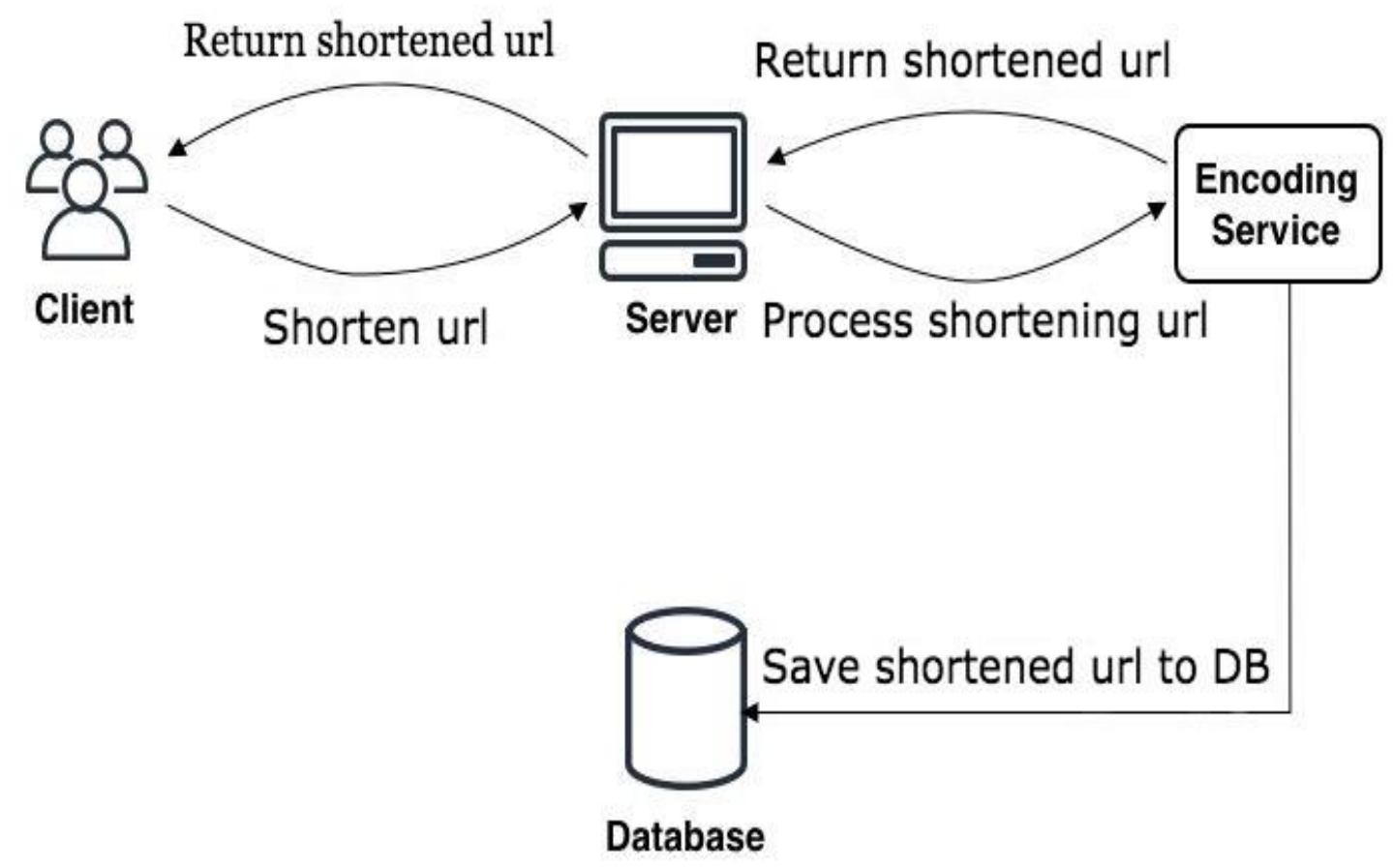

Fig.1. Architecture of URL shortening service

Figure 1 is showing how the data flows from one entity to another while carrying out the operations. The pipes are arrow marks and filters are entities like server, client, etc.

\subsection{Flask Framework for web development}

Flask is an API of Python, we used this framework for building the URL shortening application. It was developed by Armin Ronacher. As this framework is easier to learn and also has a collection of modules and libraries. This framework is based on WSGI (Web Server Gateway Interface) and Jinja2 [14-18]. There is no need of implementing the lowlevel code here. We have used Werkzeug and WSGI library. This works along with flask and it doesn't enforce any dependencies. It is up to us, the developers to choose the handling of requests, template engine and other necessary works. The flask framework looks for HTML files and these HTML files should be stored in a folder called templates. When python code is executed the variables, code, etc are loaded, before sending the template over. The Flask framework is a lightweight micro-framework. Thus it is more than suitable for creating this kind of web application.

\subsection{SQLite database engine for the data related operations}

SQLite database engine has many advantages for application which rely on server-less configuration. It gives the implementation of auto-configuration. Whatever the tables, views, triggers that we create using the application are stored in the ordinary disk files. This engine does not need any separate server process as it is an embedded SQL database 
engine. As there is no setup procedure and no configurations regarding the server protocols, there is also no need of prior instalment procedure. The programs that are execute or the processes that run on the device directly interact with the disk files for reading and writing operations. The data will be stored and can be retrieved easily even if there is a power failure. The main advantage is that any program that is able to access the disk on the computer can use this SQLite database. No separate server process even for troubleshooting.

\section{Implementing of Base62 Encoding Algorithm}

The Base62 encoding is usually used in URL Shortening. The reason behind using Base 62 encoding is because it has 62 characters, 26 upper case letters from A to Z, 26 lower case letters, 10 numbers from 0 to 9 , it is similar to base 64 , except it excludes,$+ /$, and $=$. We will be using python programming language for implementing the algorithm. There will be a unique ID generated for the long URL that the user provides to get the shortened URL. We will use this unique id to create the encoded string using base 62 algorithms. Let us consider an example and see the working of algorithm. Suppose the unique id is 200 and this is the decimal form, base62 encoding will be done using modulo operation as follows:

200 will lead to $3^{*} 62^{\wedge} 1+14^{*} 62^{\wedge} 0$, the next step is, we will convert these digits $[3,14]$ to base62 representation, resulting in a string. This would lead to "do" as base $62[3]=\mathrm{d}$ and base $62[14]=0$.

\section{Algorithm (a): base62 encoding}

Begin

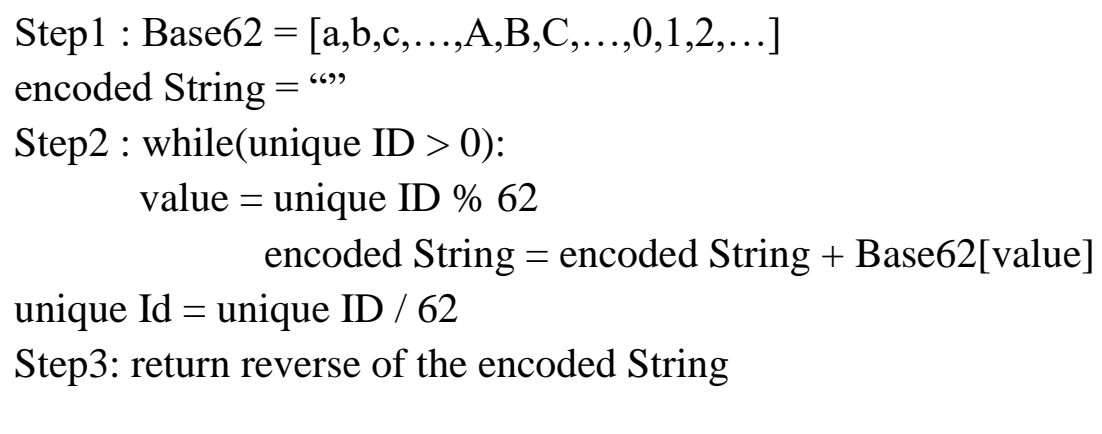




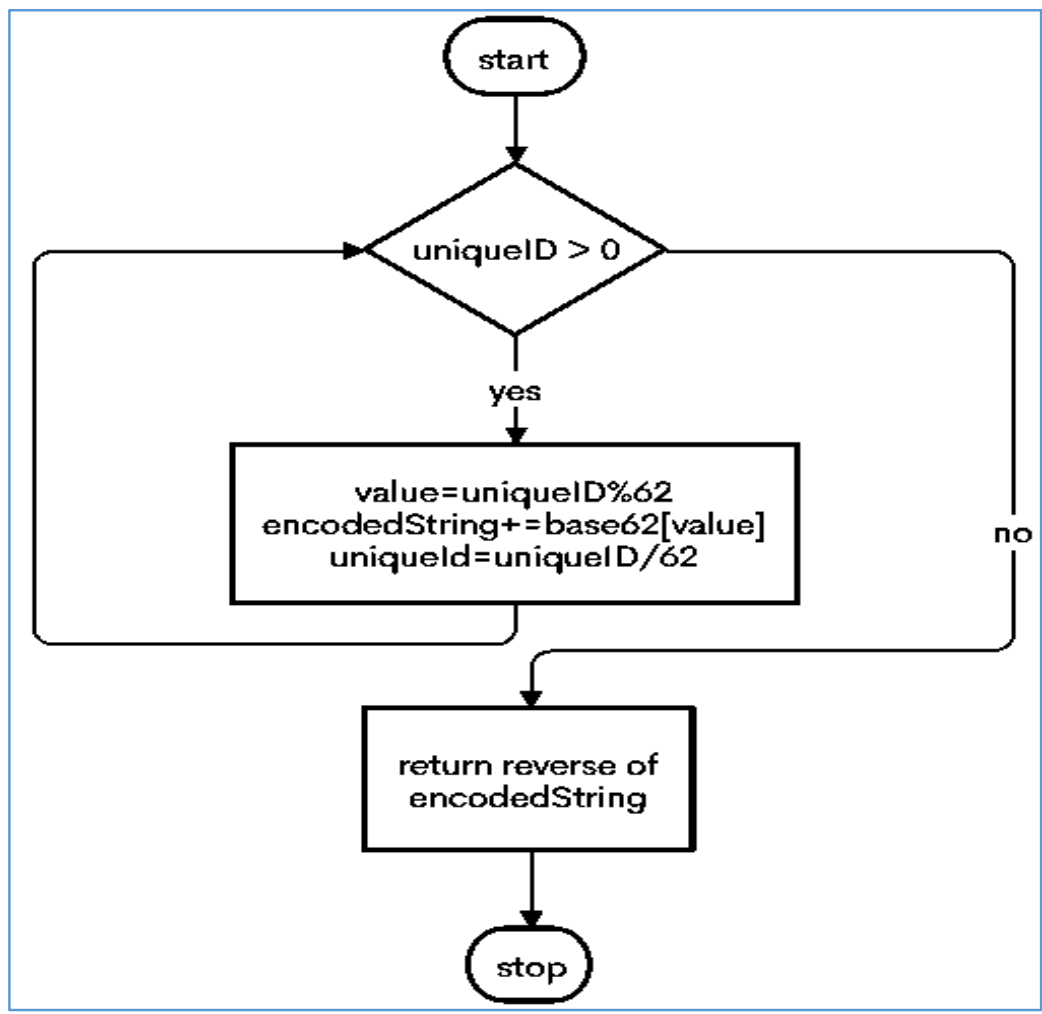

Fig.2. Flowchart for base62 algorithm encoding.

The components of URL shortening service includes URL validation, URL generation, URL redirection, Stats Of URL. Figure 2 shows the process flow of base62 encoding algorithm.

\subsection{URL validation}

The application will be checking if the submitted URL is long enough to be shortened or not; because not all URLs are long. The short URL that we generate will not exceed more than 40 characters and meeting the limitations set by different social networking platforms. We also check whether the provided input is not just a simple long text but a valid URL with the basic criteria of starting with "https://".

\section{Algorithm (1): URL Validation}

Input: URL

Output: Can be shortened or not

Begin

Step1: Enter URL

Step2: If the URL meets basic criteria Then

Go to step3

Else

Enter valid URL

Exit;

Step3: If length $(\mathrm{URL})>40$ Then

Go to Algorithm (2) 
URL needs to be shortened

Else

Use the same URL

Exit;

End If

End

\section{2 URL Generation}

After the validation process, the long URL is stored in the database and a unique identifier is created using which we will be creating the encoded string using our base 62 encoding algorithm and the user is provided the shortened URL which is then encoded string combined with our custom domain.

\section{Algorithm (2): URL Generation \\ Input : URL}

Output: Shortened URL

Begin

Step1: Enter the URL

Step2: If the URL already exists in the database Then

Get the uniqueID of URL and goto Step3

Else

generate the unique id for the URL,

store the URL in the database

and goto next step

End If

Step3: By using the process mentioned in

Algorithm(a) for the generation of an encoded string of length 7.

Step4 : The generated encode string is combined

Is combined with custom domain

http://<custom-domain-name>/ur/<encoded-string>

Step5: return the shortened URL

End

\subsection{URL Redirection :}

Here, the user enters the shortened URL provided by our service in any of the search engines. As the shortened URL contains the domain name, the database related to that particular domain will be checked for the shortened URL and the respective original long URL is retrieved and the user is redirected to that intended web address.

The shortened URLs lifespan is limited to some time and if their lifetime expires they are not stored in the database and become inaccessible and they are referred to as dead links and the database is also checked for the activeness of the URL, if it is dead then a page not found error will be displayed to the user. 


\section{Algorithm (3): URL Redirection}

Input: Shortened URL

Output: Redirecting to the original web address

Begin

Step1: Enter the shortened URL

Step2: If the unique ID of the shortened URL is in DB

Then

retrieve the long URL web address

take the user to that intended web address

Else

Display page not found an error

$$
\text { Exit; }
$$

\section{End If}

\section{Result Analysis}

When a user is registered with a URL shortening service, he can keep track of the stats of the shortened URLs that he has created using this application. The service provides the user with the information of the date and time of the creation of the short URL and also the number of clicks made to that particular short URL. When the user visits the application to check the stats of created short URLs he visits the dashboard, and he can also edit the shortened URL if the lifetime of that URL is expired. Through the stats, the user can check how many visitors are coming to their site and can get a daily breakdown. This tracking of the clicks made to the URLs is for digital marketers for tracking large campaigns.

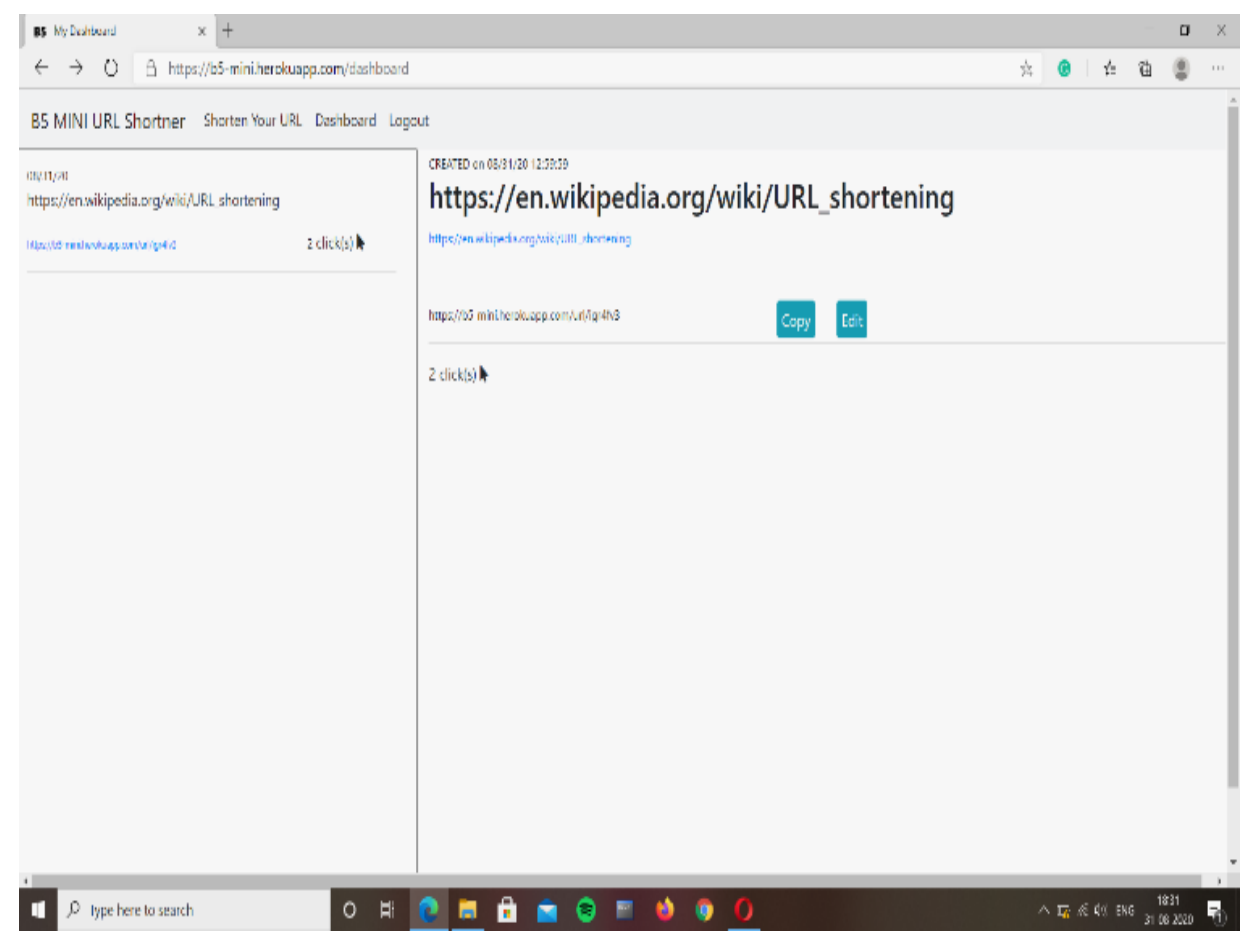

Fig.3. The interface of the URL shortening service showing the stats of created shortened URL by the user. 
Table 1 Capacity estimations and constraints.

\begin{tabular}{ll}
\hline Constraint & Estimation \\
\hline Incoming URLs & $100 / \mathrm{s}$ \\
The URL Redirections to be made & $10 \mathrm{~K} / \mathrm{s}$ \\
Incoming Data to the Service & $50 \mathrm{~KB} / \mathrm{s}$ \\
Outgoing request & $5 \mathrm{MB} / \mathrm{s}$ \\
Memory for storing frequent URLs & $85 \mathrm{~GB}$ \\
\hline
\end{tabular}

In table 1 we have shown the expected capacity estimations and constraints data which show the required elements necessary for the maintenance of the URL shortening service and its related data to maintain smooth user experience. Since we are planning to store a huge number of URLs.

\section{Conclusion}

In this paper, we have provided a brief overview of how the URL shortening service works. The proposed shortening has features such as, Putting the domain name in the shortened URL that gives the user trust to use the service, providing the user with the option of editing the URL when needed, maintaining the record of shortened URLs and their data. The application also provides authentication of the user by hashing the password and confirming it with the one stored in the database, thus maintaining the application secure from the people who are not registered. The advantages of using a URL shortening service includes attractiveness to the URL which gives the user a clutter-free view of the content while seeing any webpage containing the short URL, ease of sharing on different platforms, and avoiding the problem of breakage of URLs when shared traditionally, ease of maintaining as we are having a shorter version of URL, etc. The service also comes with few disadvantages like privacy breach, dead links that is they become inaccessible after some time, but here in our application, we are giving the user a way to check the creation details of the URL and also allowing him to edit it when needed. We can get the best of the service when used properly and ethically.

\section{References}

[1] K. Thomas, C. Grier, J. Ma, V. Paxon, and D. Song, "Design and evaluation of a real-time url spam filtering service," in Proceedings of the 2011 IEEE Symposium on Security and Privacy, ser. SP'11, 2011.

[2] Demetris Antoniades, Iasonas Polakis, and Georgios Kontaxis; "we.b: The web of short URLs", Hyderabad, India, IEEE, March 28-April 1, 2011.

[3] Sophie Le Page, Guy Vincent Jourdan, Gregor V. Bochmann, Jason Flood, and Iosif Viorel Onut. Using URL shorteners to compare phishing and malware attacks. In Proc. of eCrime, pages 1-13, 2018. 
[4] P. Iyappan, R. Muthaiya Ram, R. Barani, S. Kumara krishnan "Enhanced Shortened Uniform Resource Locator Bases on Phishing and Malware Detection Algorithm-A Secure Model", 2019 IEEE International Conference on System, Computation, Automation, and Networking (ICSCAN), , Pondicherry, India, IEEE, 30 March 2019.

[5] L.Jie and S.Bo, "An effective method for translating HTML/XHTML into WML", Journal of Bejing Technology and Business University (Natural Science Edition), vol. 24, no. 6, pp. 45-48, IEEE, Nov 2006.

[6] M. Fowler, "Refactoring: Improving the design of existing code", IEEE, 11th European Conference, 1997.

[7] D. Wang, S. B. Navathe, L. Liu, D. Irani, A. Tamersoy and C. Pu, "Click traffic analysis of short URL spam on twitter", Collaborative Computing: Networking Applications and Worksharing (Collaborate-com), pp. 250-259, IEEE, March, 2013.

[8] Cesare Pautasso, Olaf Zimmermann, and Frank Leymann, "Restful web services vs. big'web service: making the right architectural decision", Proceedings of the IEEE 17th international conference on World Wide Web, 2008.

[9] A. Ramachandran, N. Feamster, and S. Vempala, "Filtering spam with behavioral blacklisting," in Proceedings of the 14th ACM Conference on Computer and Communications Security, 2007.

[10] K. Lee, J. Caverlee, and S. Webb, "Uncovering social spammers: social honeypots+ machine learning," in Proceeding of the International ACM SIGIR Conference on Research and Development in Information Retrieval, 2010.

[11] A. Neumann, J. Barnickel, and U. Meyer, "Security and privacy implications of url shortening services," in Proceedings of the Workshop on Web 2.0 Security and Privacy, 2010.

[12] Q. Cui, G.-V. Jourdan, G. V. Bochmann, R. Couturier, and I.-V. Onut, “Tracking phishing attacks over time, " in Proceedings of the 26th International Conference on World Wide Web. International World Wide Web Conferences Steering Committee, 2017, pp. 667-676.

[13] R. K. Nepali and Y. Wang, "You look suspicious!!: Leveraging visible attributes to classify malicious short urls on twitter, ” in System Sciences (HICSS), 2016 49th Hawaii International Conference on. IEEE, 2016, pp. 2648-2655.

[14] APWG, “Workshop agenda 2017,” https://www.apwg.eu/apwg-events/ ecrime2017eu/agenda.

[15] Kumar, A., S.P. Ghrera, and V. Tyagi, Modified buyer seller watermarking protocol based on discrete wavelet transform and principal component analysis. 2015.

[16] Kumar, A., S.P. Ghrera, and V. Tyagi, An ID-based Secure and Flexible Buyerseller Watermarking Protocol for Copyright Protection. 2017.

[17] Kumar, A., S.P. Ghrera, and V. Tyagi, Implementation of wavelet based modified buyer-seller watermarking protocol (BSWP). 2014.

[18] Kumar, A., Design of secure image fusion technique using cloud for privacypreserving and copyright protection. International Journal of Cloud Applications and Computing (IJCAC), 2019. 9(3): p. 22-36. 